\title{
TOXICITY AND REPELLENCY EFFECT OF SOME INDIGENOUS PLANT EXTRACTS AGAINST LESSER GRAIN BORER, RHYZOPERTHA DOMINICA (F.) (COLEOPTERA: BOSTRYCHIDAE)
}

\author{
Md Arifuzzaman, Md Adnan Al Bachchu*, Most. Omme Kulsum, Roushan Ara \\ Department of Entomology, Hajee Mohammad Danesh Science and Technology University, \\ Dinajpur, Bangladesh
}

\begin{abstract}
Context: Insect bio assay and repellency test can play a vital role in special environmental conditions. Objectives: To screen out the insecticidal potency of some plant extracts to control the adult lesser grain borer by insect bioassay and repellency tests.

Materials and Methods: Toxicity test of five indigenous plant extracts with three concentrations were conducted against Rhizopertha dominica. Insect mortality was recorded at 24, 48, and 72 HAT. For residual toxicity test, insect mortality was recorded at 1, 2, 7, 15 and 21 DAT. The repellent activities were evaluated using the filter paper impregnation method and the data were counted at hourly intervals up to $6^{\text {th }}$ hour. In all cases ten insects per replication were tested and each treatment was replicated thrice. The collected data were statistically analyzed.

Results: Among the tested plant extracts, neem showed the highest toxic and repellent effects against the lesser grain borer. All the doses applied had direct toxicity, residual and repellency effects while $8 \%$ dose showed the highest response. The order of toxicity was found as neem $>$ biskatali $>$ karabi $>$ akanda $>$ ata. Mortality percentages were directly proportional to the time after treatment.

Conclusion: This study proved that the leaf extract of indigenous plants like neem, biskatali, karabi ata and akanda can be used to protect stored grain pests.
\end{abstract}

Key words: Mortality, repellency, residual effect, petroleum ether, Rhizopertha dominica.

\section{Introduction}

Losses due to insect infestation are the most serious problem in storage, particularly in villages and towns of developing countries like Bangladesh. Storage loss may also be significant in developing countries (70\%) (Kavita 2004). It has been estimated that about $15-20 \%$ of the world agricultural production is lost every year due to insect infestation (Wright 1985). In Bangladesh, the annual grain losses cost over taka 100 cores (Alam 1971) whereas in India losses caused by insects accounted for 6.5\% of stored grain (Kumar 2009). The climate and storage conditions, especially in the tropics, are often highly favorable for insect growth and development (Jacobson 2004). Their attacks reduce both the quantity and quality of stored seed. Sometimes moulds grow in the insect infested food grain and these moulds produce a chemical substance called aflatoxin which is reported to be associated with the liver cancer of human being (Singh 1983). Rhizopertha dominica Fab. (Coleptera: Bostrichidae) is the most common and injurious to stored grains having an important position among the storage pests. It is a field-to-store pest and cause economic damage (Adedire 2001). Both the adults and grubs causes' serious damage to stored grains and stored products and adult beetles are more harmful which destroy healthy grains and reduced them to frass. They destroy far more than they consume.

*Corresponding author Email: adnan_hstu@yahoo.com, aabachchu1975@gmail.com 
Synthetic chemical pesticides have been used for many years to control stored grain pests (Salem et al. 2007). Fumigation of stored food grains with toxic gases is effective but not applicable at the farm level because the storage structures are not airtight. Furthermore, control of insects by insecticides has serious drawbacks, such as the toxic residues on stored grains, development of resistance by target species, pest resurgence and lethal effects on non-target organisms in addition to direct toxicity to users and health hazard (Adedire and Lajide 2003, Adedire et al. 2011, lleke and Oni 2011, Udo, 2011, lleke and Olotuah 2012, lleke and Bulus 2012). This situation indicates the need for safe but effective, biodegradable pesticides with no toxic effects on non-target organisms for pest control in storage. Recently, there is a steady increase in the use of indigenous plant products as a cheaper and ecologically safer means of protecting stored products against infestation by insects (Ashamo and Odeyemi 2001, Oni and lleke 2008, Akinkurolere et al. 2009, lleke et al. 2012) and this lead to the present study.

\section{Materials and Methods \\ Preparation of plant extracts}

Fresh leaves of ata (Annona reticulate L.), karabi (Nerium oleander L.), neem (Azadirachta indica L.), biskatali (Polygonum hydropiper L.) and akanda (Calotropis gigantean L.) leaves extracts were used against $R$. dominica in the laboratory, Entomology Department, Hajee Mohammad Danesh Science and Technology University, Dinajpur during April to December 2012. They were collected around the HSTU campus. They were washed in running water and kept in laboratory for 7 days air drying. After drying they were made powder separately by an electric grinder. The extracts were prepared according to (Chitra et al. 1993) with minor modifications. For making extracts, $100 \mathrm{~g}$ of different plant powders were dissolved in $300 \mathrm{ml}$ of petroleum ether solvent and stirred for $30 \mathrm{~min}$. in a magnetic stirrer. The mixture was allowed to stand for 72 hours and shaking several intervals. It was filtered through a filter paper (Whatman no. 1) and to evaporate the solvents. The condensed extracts were preserved in tightly corked-labeled bottles and stored in a refrigerator until their further use.

\section{Collection of wheat grains}

Healthy wheat grains, Triticum aestivum (L.) were purchased from the local market of Dinajpur town, cleaned thoroughly and sun dried. The grains were cooled at 8-10\% moisture level and stored at room temperature in air tight plastic bag for experimental use.

\section{Mass rearing of Rhyzopertha dominica}

$R$. dominica were collected from the naturally infested wheat grains from the local market of Dinajpur and was mass reared in the laboratory at ambient room temperature $\left(28 \pm 0.5^{\circ} \mathrm{C}\right)$ in glass jars $(47 \mathrm{~cm}$ height $\times 4$ $\mathrm{cm}$ dia). Approximately 200 adults were released in each glass jar containing $500 \mathrm{~g}$ of wheat grains and the mouth was closed with a piece of cloth fastened with rubber band to prevent contamination and escape of insect. After oviposition, the adults were separated from the grains by sieving and seeds along with eggs were left in the container for emergence of next generation. The newly emerged adults (1-7- days- old) were collected and again allowed for oviposition with new grains in different containers to maintain a stock culture of the test insect. The process was containing for getting enough pest throughout the study.

\section{Evaluation of toxicity of different plant extracts}

Toxicity test were conducted according to (Talukdar and Howse 1993) with minor modifications. The extracted materials were weighed and dissolved in petroleum for making different concentration (4.0, 6.0 and $8.0 \%$ along with control). Pilot experiments were done to obtain the appropriate dose. Before applying extracts to the thorax of the insect, 10 minutes chilling were done with $4^{\circ} \mathrm{C}$ in refrigerator. Then $1 \mu \mathrm{l}$ of prepared solution was applied to the dorsal surface of each insect using a micropipette (volume digital micropipets, bio-rad, India). Ten insects per replication were treated and each treatment was replicated 
thrice. In addition, the same numbers of insects with petroleum ether solvent only were treated as control. After treatment, the insects were transferred into petridishes $(9 \mathrm{~cm}$ diameter). Mortality was recorded after 24, 48, and 72 hours treatment (HAT) (Talukdar and Howse 1993). The data were corrected according to Abott's (1987) formula.

\section{Determination of residual effect}

Three different concentrations of each plant-extract were prepared with the petroleum ether solvent. Then 1 $\mathrm{ml}$ of prepared solution was applied to $50 \mathrm{~g}$ wheat and mixed properly. After 10 minutes air-dried five pairs (1 - day- old) insects were released into the pot containing plant extracts treated wheat grain and then pot was covered with perforated lid. Three replications were maintained for each of the concentration of the individual plant extracts along with control. All treated pots were kept at ambient temperature $\left(28 \pm 0.5^{\circ} \mathrm{C}\right)$ in the laboratory. Mortality was recorded at 1, 2, 7, 15 and 21 DAT (days after treatment).

\section{Detection of repellency}

First of all, the Whatman No. 1 filter papers were cut into two half. With the help of a pipette $1 \mathrm{ml}$ solution of each plant extract was applied to one half of the filter paper and only petroleum ether solvent was used for another half as control. The treated half and control half was then air-dried. Ten insects were released at the centre of each petridish with a cover. For each plant extract and each dose three replications were used. The insect present on each portion were counted at hourly intervals up to $6^{\text {th }}$ hour. The data were expressed as percentage repulsion (\%PR) by the following formula (Talukdar and Howse 1994):

$\% \mathrm{PR}=(\mathrm{Nc}-50) \times 2$

Where, $\% \mathrm{PR}=$ percentage repulsion, $\mathrm{N}_{\mathrm{c}}=$ percentage of insects present in the control half.

Positive $(+)$ values expressed repellency and negative (-) values attractency. The average values were categorized according to the following scale (McDonald et al. 1970)

\begin{tabular}{|c|l|c|l|}
\hline Class & & Class & \multicolumn{1}{|c|}{ Repellency (\%) } \\
\hline 0 & $>0.01$ to 0.1 & III & 40.1 to 60 \\
\hline I & 0.1 to 20 & IV & 60.1 to 80 \\
\hline II & 20.1 to 40 & V & 80.1 to 100 \\
\hline
\end{tabular}

Statistical analysis

The collected data were statistically analyzed by completely randomized design (CRD) using MSTAT statistical software. The treatment mean values were adjusted by Duncun's New Multiple Range Test (DMRT) and mortality data subjected to probit analysis.

\section{Results and Discussion}

Effects of direct toxicity against lesser grain borer

Mortality was differed significantly $(p<0.001)$ among all the concentration level at different time interval of different plant extracts. All plant extracts except Kkarabi achieved hundred percent mortality at 72 HAT in $8.0 \%$ concentrations. Average mortality percentage of indicated that neem leaf extract $(98.90 \%)$ possessed the highest toxic effect followed by biskatali (94.43\%) and karabi (56.63\%) possessed the lowest toxic effect (Table 1). The order of toxicity of five plant extracts were found as neem $>$ biskatali $>$ akanda $>$ ata $>$ karabi. The above findings revealed that all tested plants extracts are toxic against lesser grain borer and the neem plant extracts showed the highest toxic effect. This finding agreed with lleke and Bulus (2012) who work with the response of $R$. dominica to powders and extracts of Azadirachta indica and Piper guineense seeds and showed that adult mortality increased both concentration of powders and extracts. 
Table 1. Interaction effects of plant extract and concentration on lesser grain borer at different HAT (Hours after treatment).

\begin{tabular}{|c|c|c|c|c|c|}
\hline \multirow{2}{*}{$\begin{array}{l}\text { Plant extracts } \\
\text { used }\end{array}$} & \multirow[t]{2}{*}{ Concentrations (\%) } & \multicolumn{4}{|c|}{$\%$ Insect mortality (HAT) } \\
\hline & & 24 & 48 & 72 & Average \\
\hline \multirow{4}{*}{ Neem } & 0.0 & $0.00 \mathrm{~h}$ & $0.00 \mathrm{~g}$ & $0.00 \mathrm{i}$ & 0.00 \\
\hline & 4.0 & $40.00 d$ & $76.67 \mathrm{bc}$ & $80.00 \mathrm{bc}$ & 65.57 \\
\hline & 6.0 & $56.67 c$ & $90.00 \mathrm{ab}$ & $100.0 \mathrm{a}$ & 82.23 \\
\hline & 8.0 & $96.67 a$ & $100.0 \mathrm{a}$ & $100.0 \mathrm{a}$ & 98.90 \\
\hline \multirow{4}{*}{ Biskatali } & 0.0 & $0.00 \mathrm{~h}$ & $0.00 \mathrm{~g}$ & $0.00 \mathrm{i}$ & 0.00 \\
\hline & 4.0 & $16.67 \mathrm{fg}$ & $23.33 f$ & 40.00ef & 26.67 \\
\hline & 6.0 & $40.00 d$ & $76.67 \mathrm{bc}$ & $90.00 \mathrm{ab}$ & 68.90 \\
\hline & 8.0 & $83.33 b$ & $100.0 \mathrm{a}$ & $100.0 \mathrm{a}$ & 94.43 \\
\hline \multirow{4}{*}{ Karabi } & 0.0 & $0.00 \mathrm{~h}$ & $0.00 \mathrm{~g}$ & $0.00 \mathrm{i}$ & 0.00 \\
\hline & 4.0 & 3.33gh & $3.33 \mathrm{~g}$ & $20.00 \mathrm{~h}$ & 8.90 \\
\hline & 6.0 & $16.67 \mathrm{fg}$ & $36.67 \mathrm{ef}$ & 46.67de & 33.37 \\
\hline & 8.0 & 33.33de & $60.00 \mathrm{~d}$ & $76.67 c$ & 56.63 \\
\hline \multirow{4}{*}{ Akanda } & 0.0 & $0.00 \mathrm{~h}$ & $0.00 \mathrm{~g}$ & $0.00 i$ & 0.00 \\
\hline & 4.0 & 23.33ef & $23.33 f$ & $26.67 \mathrm{gh}$ & 24.43 \\
\hline & 6.0 & 36.67de & 63.33cd & $73.33 c$ & 57.77 \\
\hline & 8.0 & $76.67 \mathrm{~b}$ & $96.67 a$ & $100.0 \mathrm{a}$ & 91.10 \\
\hline \multirow{4}{*}{ Ata } & 0.0 & $0.00 \mathrm{~h}$ & $0.00 \mathrm{~g}$ & $0.00 \mathrm{i}$ & 0.00 \\
\hline & 4.0 & $16.67 \mathrm{fg}$ & $23.33 f$ & $33.33 \mathrm{fg}$ & 24.43 \\
\hline & 6.0 & 33.33de & $43.33 \mathrm{e}$ & $56.67 d$ & 44.47 \\
\hline & 8.0 & $60.00 \mathrm{c}$ & $86.67 a b$ & $100.0 \mathrm{a}$ & 82.20 \\
\hline \multirow{3}{*}{$\begin{array}{l}\text { P- value } \\
\text { LSD } \\
\text { CV (\%) }\end{array}$} & & 0.0001 & 0.0001 & 0.0001 & - \\
\hline & & 13.24 & 14.98 & 11.98 & - \\
\hline & & 4.29 & 4.06 & 3.90 & - \\
\hline
\end{tabular}

HAT = Hours after treatment, Mean followed by column the same letter(s) did not differ significantly at 5\% level by DMRT .

Probit analysis for direct toxicity

The LD50 values of neem $(6.958 \mu \mathrm{g})$, biskatali $(8.444 \mu \mathrm{g})$, karabi $(9.191 \mu \mathrm{g})$, akanda $(10.589 \mu \mathrm{g})$ and ata (14.522 $\mu \mathrm{g}$ ) at $24 \mathrm{HAT}$ (Table 2) indicated that neem leaf extract was found to be highly toxic. Similarly, neem leaf extract maintain its highest toxicity at 48 and 72 HAT. The results obtained in this probit study showed that all the tested plants would be more or less effective for controlling lesser grain borer but neem was most effective. The lowest $\mathrm{LD}_{50}$ values of neem plant extract indicated that the highest toxic effects against lesser grain borer to suppress their population growth in treated wheat grains. Some researchers who had earlier evaluated $A$. indica powder and extract as botanical insecticides and grains protectant had found them to be effective against $S$. zeamais and $C$. maculatus (Onu and Baba 2003, lleke and Oni 2011, lleke and Bulus 2012). The toxicity of neem to stored products insects has been attributed by various authors to the presence of many chemical ingredients such as triterpenoids, which includes azadirachtin, salanin, meliantriol (Mbailao et al. 2006, lleke and Oni 2011).

\section{Effect of residual toxicity against lesser grain borer}

The effects of residual toxic of plant extracts, doses and time revealed that neem plant extract possessed the highest residual effect (average mortality, 98.97\%) followed by biskatali (average mortality $95.20 \%$ ) against lesser grain borer at maximum dose $(8.0 \%)$. Mortality percentages were differed significantly between plant extracts and doses (Table 3). The toxic effect of five plant extracts was: neem $>$ biskatali $>$ karabi $>$ akanda $>$ ata. 
Table 2. Relative toxicity (by probit analysis) of different plant extracts treated against lesser grain borer at 24,48 and 72 HAT.

\begin{tabular}{|c|c|c|c|c|}
\hline Name of the plant extracts & $\begin{array}{l}\text { No. of insect } \\
\text { used }\end{array}$ & LD 50 values $(\mu \mathrm{g})$ & $95 \%$ fiducially limits & $Y^{2}$ values \\
\hline \multicolumn{5}{|c|}{$24 \mathrm{HAT}$} \\
\hline Neem & 90 & 6.958 & $3.646-13.278$ & 0.0055 \\
\hline Biskatali & 90 & 8.444 & $4.251-16.770$ & 0.0514 \\
\hline Karabi & 90 & 9.191 & $4.262-19.822$ & 0.0943 \\
\hline Akanda & 90 & 10.589 & $4.827-23.226$ & 0.1735 \\
\hline Ata & 90 & 14.522 & $3.644-57.870$ & 0.3752 \\
\hline \multicolumn{5}{|c|}{$48 \mathrm{HAT}$} \\
\hline Neem & 90 & 1.448 & $0.021-97.257$ & 0.0439 \\
\hline Biskatali & 90 & 3.709 & $1.716-8.013$ & 0.0015 \\
\hline Karabi & 90 & 5.771 & $4.4933-7.412$ & 0.2081 \\
\hline Akanda & 90 & 6.349 & $4.947-8.148$ & $0.0476 \mathrm{E}-04$ \\
\hline Ata & 90 & 9.342 & $5.466-15.966$ & 0.1611 \\
\hline \multicolumn{5}{|c|}{72 HAT } \\
\hline Neem & 90 & 2.744 & $1.630-4.755$ & 0.3102 \\
\hline Biskatali & 90 & 2.784 & $1.449-5.195$ & 0.0730 \\
\hline Karabi & 90 & 3.432 & $2.131-5.528$ & 0.0339 \\
\hline Akanda & 90 & 4.127 & $2.940-5.794$ & 0.1356 \\
\hline Ata & 90 & 5.924 & $4.989-7.034$ & 0.0116 \\
\hline
\end{tabular}

HAT= Hour after treatment, Values were based on three concentrations, three replications of 10 insects each, $X^{2}=$ Goodness of fit, the tabulated value of $\mathrm{X}^{2}$ is 5.99 ( $\mathrm{d}$. $\mathrm{f}=2$ at $5 \%$ level).

Table 3. Interaction effects of plant extract and concentration on the lesser grain borer at different DAT (Days after treatment).

\begin{tabular}{|c|c|c|c|c|c|c|c|}
\hline \multirow{2}{*}{$\begin{array}{l}\text { Plant extracts } \\
\text { used }\end{array}$} & \multirow{2}{*}{$\begin{array}{l}\text { Concentratio } \\
\text { ns (\%) }\end{array}$} & \multicolumn{6}{|c|}{$\%$ Insect mortality (DAT) } \\
\hline & & $1 \mathrm{DAT}$ & 2 DAT & 7 DAT & $15 \mathrm{DAT}$ & $21 \mathrm{DAT}$ & Average \\
\hline \multirow{4}{*}{ Neem } & 0.0 & $0.00 \mathrm{~h}$ & $0.00 f$ & $0.00 \mathrm{~g}$ & $0.00 \mathrm{i}$ & $0.00 \mathrm{i}$ & 0.00 \\
\hline & 4.0 & $40.00 \mathrm{~d}$ & $56.67 d$ & $76.67 \mathrm{bc}$ & $78.33 b c$ & $80.00 \mathrm{bc}$ & 66.33 \\
\hline & 6.0 & $55.67 c$ & $75.33 c$ & $90.00 \mathrm{ab}$ & $96.00 a b$ & $100.0 \mathrm{a}$ & 83.40 \\
\hline & 8.0 & $94.33 a$ & $95.67 a$ & $100.0 \mathrm{a}$ & $100.0 \mathrm{a}$ & $100.0 \mathrm{a}$ & 98.97 \\
\hline \multirow{4}{*}{ Biskatali } & 0.0 & $0.00 \mathrm{~h}$ & $0.00 f$ & $0.00 \mathrm{~g}$ & $0.00 f$ & $0.00 \mathrm{i}$ & 0.00 \\
\hline & 4.0 & $15.67 f g$ & $20.33 e$ & $23.33 f$ & $29.67 \mathrm{~g}$ & 55.00ef & 28.80 \\
\hline & 6.0 & $38.00 \mathrm{~d}$ & $46.33 c$ & $76.67 \mathrm{bc}$ & $85.67 a$ & $93.00 \mathrm{ab}$ & 67.93 \\
\hline & 8.0 & $83.33 b$ & $92.67 \mathrm{~b}$ & $100.0 \mathrm{a}$ & $100.0 \mathrm{a}$ & $100.0 \mathrm{a}$ & 95.20 \\
\hline \multirow{4}{*}{ Karabi } & 0.0 & $0.00 \mathrm{~h}$ & $0.00 f$ & $0.00 \mathrm{~g}$ & $0.00 f$ & $0.00 \mathrm{i}$ & 0.00 \\
\hline & 4.0 & 20.33ef & $22.33 e$ & $23.33 f$ & $25.33 \mathrm{ef}$ & $36.67 \mathrm{gh}$ & 25.60 \\
\hline & 6.0 & 35.33de & $46.67 \mathrm{~cd}$ & $63.33 \mathrm{~cd}$ & $69.67 c$ & $79.33 c$ & 58.87 \\
\hline & 8.0 & $74.67 \mathrm{~b}$ & $88.67 b$ & $96.67 a$ & $100.0 \mathrm{a}$ & $100.0 \mathrm{a}$ & 92.00 \\
\hline \multirow{4}{*}{ Akanda } & 0.0 & $0.00 \mathrm{~h}$ & $0.00 f$ & $0.00 \mathrm{~g}$ & $0.00 f$ & $0.00 \mathrm{i}$ & 0.00 \\
\hline & 4.0 & $13.67 \mathrm{fg}$ & $21.67 \mathrm{e}$ & $22.33 f$ & $34.67 \mathrm{e}$ & $35.33 f g$ & 25.53 \\
\hline & 6.0 & 30.33de & $40.67 c$ & $48.33 e$ & $54.67 \mathrm{c}$ & $59.67 d$ & 46.73 \\
\hline & 8.0 & $58.33 c$ & $76.67 \mathrm{~b}$ & 89.67ab & $95.33 \mathrm{bc}$ & $100.0 \mathrm{a}$ & 84.00 \\
\hline \multirow{4}{*}{ Ata } & 0.0 & $0.00 \mathrm{~h}$ & $0.00 \mathrm{f}$ & $0.00 \mathrm{~g}$ & $0.00 f$ & $0.00 \mathrm{i}$ & 0.00 \\
\hline & 4.0 & 7.33gh & $9.33 f$ & $12.33 \mathrm{~g}$ & $16.67 f$ & $20.00 \mathrm{~h}$ & 13.13 \\
\hline & 6.0 & $16.33 \mathrm{fg}$ & 25.67de & $39.67 \mathrm{ef}$ & 45.33de & 49.67de & 35.33 \\
\hline & 8.0 & $35.67 \mathrm{de}$ & $49.67 c$ & $66.00 \mathrm{~d}$ & $70.00 \mathrm{c}$ & $80.67 c$ & 60.40 \\
\hline \multicolumn{2}{|l|}{$P$-value } & 0.0001 & 0.0001 & 0.0001 & 0.0001 & 0.0001 & - \\
\hline \multicolumn{2}{|l|}{ LSD } & 14.24 & 14.98 & 15.98 & 13.98 & 12.98 & - \\
\hline \multicolumn{2}{|l|}{ CV (\%) } & 5.29 & 6.20 & 7.06 & 4.65 & 3.90 & - \\
\hline
\end{tabular}

DAT = Days after treatment, Mean followed by the same letter(s) did not differ significantly at $5 \%$ level by DMRT. 


\section{Probit analysis for residual toxicity}

The $L_{50}$ values of neem $(18.543 \mu \mathrm{g})$, biskatali $(29.817 \mu \mathrm{g})$, karabi $(30.937 \mu \mathrm{g})$, akanda $(34.883 \mu \mathrm{g})$ and ata $(31.204 \mu \mathrm{g})$ at 1 DAT (Table 4) indicated that neem plant extracts possessed the highest toxicity while lowest in akanda against the test insect. Similarly, neem plant extract maintained its highest toxicity at 2, 7, 15 and 21 DAT. The chi-square $\left(\gamma^{2}\right)$ values of different plant extracts at different hours after treatment were insignificant. It is clear that neem leaf extracts possessed the highest toxicity in controlling lesser grain borer. The compound azadirachtin may work as an Insect Growth Regulator (IGRs) interfering with ecdysone which prevents immature insects from molting (Soon and Bottrell 1994).

Table 4. Relative toxicity (by probit analysis) of different plant extracts treated against lesser grain borer at $1,2,7,15$ and 21 DAT.

\begin{tabular}{|c|c|c|c|c|}
\hline $\begin{array}{l}\text { Name of the plant } \\
\text { extracts }\end{array}$ & $\begin{array}{l}\text { No. of insect } \\
\text { used }\end{array}$ & $\begin{array}{c}\text { LC } 50 \text { values } \\
(\mu \mathrm{g})\end{array}$ & $95 \%$ fiducially limits & $\mathrm{Y}^{2}$ values \\
\hline \multicolumn{5}{|c|}{$1 \mathrm{DAT}$} \\
\hline Neem & 90 & 18.543 & $2.721-126.347$ & 0.019 \\
\hline Biskatali & 90 & 29.817 & $1.071-830.053$ & 0.051 \\
\hline Karabi & 90 & 30.937 & $1.388-689.277$ & 0.095 \\
\hline Akanda & 90 & 34.883 & $1.432-849.918$ & 0.023 \\
\hline Ata & 90 & 31.204 & $1.648-590.492$ & 0.082 \\
\hline \multicolumn{5}{|c|}{2 DAT } \\
\hline Neem & 90 & 11.446 & $3.591-36.476$ & 0.097 \\
\hline Biskatali & 90 & 12.573 & $3.974-39.774$ & 0.241 \\
\hline Karabi & 90 & 19.754 & $2.579-151.281$ & 0.214 \\
\hline Akanda & 90 & 31.204 & $1.648-590.492$ & 0.082 \\
\hline Ata & 90 & 15.259 & $4.986-246.693$ & 0.052 \\
\hline \multicolumn{5}{|c|}{7 DAT } \\
\hline Neem & 90 & 5.941 & $4.833-7.302$ & 0.378 \\
\hline Biskatali & 90 & 6.312 & $5.131-7.765$ & 0.405 \\
\hline Karabi & 90 & 8.267 & $5.983-11.421$ & 0.103 \\
\hline Akanda & 90 & 11.057 & $7.065-17.303$ & 0.238 \\
\hline Ata & 90 & 10.243 & $6.164-17.023$ & 0.339 \\
\hline \multicolumn{5}{|c|}{15 DAT } \\
\hline Neem & 90 & 4.431 & $3.580-5.482$ & 0.240 \\
\hline Biskatali & 90 & 4.508 & $3.500-5.803$ & 0.139 \\
\hline Karabi & 90 & 5.412 & $4.609-6.354$ & 0.559 \\
\hline Akanda & 90 & 8.224 & $6.663-10.149$ & 0.001 \\
\hline Ata & 90 & 6.240 & $5.325-7.312$ & 0.002 \\
\hline \multicolumn{5}{|c|}{21 DAT } \\
\hline Neem & 90 & 3.765 & $2.829-5.010$ & 0.341 \\
\hline Biskatali & 90 & 3.803 & $2.849-5.076$ & 0.217 \\
\hline Karabi & 90 & 5.259 & $3.268-5.549$ & 0.629 \\
\hline Akanda & 90 & 6.429 & $5.381-7.679$ & 0.973 \\
\hline Ata & 90 & 4.849 & $3.879-6.062$ & 0.005 \\
\hline
\end{tabular}

DAT = Day after treatment, Values were based on three concentrations, three replications of 10 insects each, $X^{2}=$ Goodness of fit, the tabulated value of $\chi^{2}$ is 5.99 ( $d . f=2$ at $5 \%$ level).

\section{Effect of repellency on lesser grain borer}

Mean repellent effect of different plant extracts in different dose level on lesser grain borer is presented in Table 5. The repellency was influenced by the concentration of extracts. The rate of the extract and the repellency class were found I to $\mathrm{V}$ in all five plant extracts. 
Table 5. Repellency effect of different plant extract and their doses on lesser grain borer at different HATs.

\begin{tabular}{|c|c|c|c|c|c|c|c|c|c|}
\hline \multirow{2}{*}{$\begin{array}{l}\text { Plant } \\
\text { extracts } \\
\text { used }\end{array}$} & \multicolumn{9}{|c|}{ Repellency rate (\%) at six different HATs } \\
\hline & $\begin{array}{l}\text { Dose } \\
(\%)\end{array}$ & $1^{\text {st }}$ hour & $2^{\text {nd }}$ hour & $3^{\text {rd }}$ hour & $4^{\text {th }}$ hour & $5^{\text {th }}$ hour & $6^{\text {th }}$ hour & Average & $\begin{array}{l}\text { Repel. } \\
\text { class }\end{array}$ \\
\hline \multirow{4}{*}{ Neem } & 0.0 & $0.00 f$ & $0.00 \mathrm{e}$ & 20.00de & $0.00 c$ & $0.00 f$ & $0.00 \mathrm{~g}$ & 3.30 & 1 \\
\hline & 4 & 33.33a-f & $26.67 \mathrm{~b}-\mathrm{e}$ & 20.00de & $26.67 \mathrm{bc}$ & $26.67 c-f$ & $26.67 \mathrm{~d}-\mathrm{g}$ & 26.67 & 4 \\
\hline & 6 & 53.33a-d & $26.67 \mathrm{~b}-\mathrm{e}$ & $33.33 \mathrm{c}-\mathrm{e}$ & $20.00 c$ & $33.33 b-f$ & $13.33 \mathrm{fg}$ & 30.03 & 4 \\
\hline & 8 & $66.67 a b$ & 53.33ab & $100.0 \mathrm{a}$ & $93.33 a$ & $86.67 a$ & $93.33 a$ & 82.20 & $\mathrm{~V}$ \\
\hline \multirow{4}{*}{ Biskatali } & 0.0 & $0.00 f$ & 20.00b-e & 20.00de & $0.00 \mathrm{c}$ & $0.00 f$ & $0.00 \mathrm{~g}$ & 6.70 & I \\
\hline & 4 & $26.67 b-f$ & $40.00 a-d$ & $40.00 \mathrm{~b}-\mathrm{e}$ & $26.67 \mathrm{bc}$ & 53.33a-d & $66.67 a-d$ & 42.20 & ш \\
\hline & 6 & $60.00 \mathrm{a}-\mathrm{c}$ & $40.00 a-d$ & $46.67 \mathrm{~b}-\mathrm{d}$ & 73.33ab & $80.00 \mathrm{a}$ & $80.00 \mathrm{ab}$ & 63.33 & IV \\
\hline & 8 & $53.33 a-d$ & 26.67b-e & 13.33de & $46.67 b c$ & $20.00 d-f$ & $33.33 c-g$ & 32.23 & 4 \\
\hline \multirow{4}{*}{ Karabi } & 0.0 & $0.00 f$ & $0.00 \mathrm{e}$ & $0.00 \mathrm{e}$ & $40.00 b c$ & $0.00 f$ & $0.00 \mathrm{~g}$ & 6.70 & 1 \\
\hline & 4 & $26.67 b-f$ & $66.67 a$ & 73.33a-c & $46.67 \mathrm{bc}$ & $60.00 \mathrm{a}-\mathrm{c}$ & $66.67 a-d$ & 56.67 & ш \\
\hline & 6 & $6.66 \mathrm{ef}$ & $60.00 \mathrm{a}$ & 46.67bcd & $40.00 \mathrm{bc}$ & 13.33ef & $20.00 \mathrm{e}-\mathrm{g}$ & 31.13 & 4 \\
\hline & 8 & $40.00 a-f$ & $46.67 a-c$ & 26.67de & $26.67 b c$ & $60.00 a-c$ & 53.33a-f & 42.20 & ш \\
\hline \multirow{4}{*}{ Akanda } & 0.0 & $0.00 f$ & $0.00 \mathrm{e}$ & $80.00 a b$ & $0.00 \mathrm{c}$ & $0.00 f$ & $20.00 \mathrm{efg}$ & 16.70 & 1 \\
\hline & 4 & 46.67a-e & $66.67 a$ & $46.67 b-d$ & $46.67 \mathrm{bc}$ & $66.67 \mathrm{ab}$ & $33.33 \mathrm{c}-\mathrm{g}$ & 51.13 & w \\
\hline & 6 & $73.33 a$ & 33.33a-e & $40.00 \mathrm{~b}-\mathrm{e}$ & $40.00 \mathrm{bc}$ & 13.33ef & 60.00a-e & 43.33 & ш \\
\hline & 8 & $33.33 a-f$ & $60.00 a$ & $33.33 \mathrm{c}-\mathrm{e}$ & $33.33 b c$ & $40.00 \mathrm{~b}-\mathrm{e}$ & $40.00 \mathrm{~b}-\mathrm{g}$ & 40.00 & ш \\
\hline \multirow{4}{*}{ Ata } & 0.0 & $20.00 c-f$ & $0.00 \mathrm{e}$ & $0.00 \mathrm{e}$ & $20.00 \mathrm{c}$ & $0.00 f$ & $0.00 \mathrm{~g}$ & 6.700 & 1 \\
\hline & 4 & $13.33 d-f$ & $20.00 \mathrm{~b}-\mathrm{e}$ & 13.33de & $33.33 b c$ & $20.00 d-f$ & $33.33 c-g$ & 22.23 & 4 \\
\hline & 6 & $20.00 c-f$ & 6.66de & 20.00de & $6.66 c$ & 40.00b-e & $66.67 a-d$ & 26.67 & 4 \\
\hline & 8 & 46.67a-e & $13.33 c-e$ & 20.00de & $33.33 b c$ & $20.00 d-f$ & 73.33a-c & 34.43 & 4 \\
\hline \multicolumn{2}{|l|}{ P-value } & 0.0867 & 0.0439 & 0.0002 & 0.0071 & 0.0002 & 0.0021 & - & \\
\hline \multicolumn{2}{|l|}{ Lsd } & 36.33 & 28.70 & 36.95 & 39.76 & 33.25 & 38.76 & - & \\
\hline \multicolumn{2}{|l|}{ CV (\%) } & 4.91 & 3.23 & 4.48 & 3.63 & 3.52 & 3.12 & - & \\
\hline \multicolumn{2}{|l|}{ SE } & 12.69 & 10.02 & 12.91 & 13.89 & 11.61 & 13.54 & - & \\
\hline
\end{tabular}

Mean followed by the same letter(s) did not differ significantly at $5 \%$ level by DMRT.

The highest mean repellency (82.20\%) was found in neem extract at 8\% dose whereas lowest (3.30\%) in control treatment. With the progress of time, the repellency effect decreased in maximum cases. Neem products repel insects, stop their feeding, inhibit reproduction and cause other interruptions (Schmutterer 1990). Jilani and Saxena (1990) observed that the repellency of compounds with low molecular weights and high volatility decreased rapidly over time.

\section{Conclusion}

The present study revealed that the highest mean repellency was observed in neem extract for lesser grain borer, $R$. dominica. This study is proved our traditional use of leaves of neem, biskatali, karabi ata and akanda to protect stored grain pests.

\section{References}

Abbott W S. 1987. A method of computing the effectiveness of an insecticide. J American Mosquito Cont Assoc 3, 302303.

Adedire C O. 2001. Pests of Stored Cereals and Pulses in Nigeria. In : Ofuya T I and Lale N E S (Eds.), Biology, Ecology and Control of Insect Pests of Stored Grains. Nigeria: Dave Collins publication. pp 59-94.

Adedire C O, Lajide L. 2003. Ability of extract of ten tropical plant species to protect maize grains against infestation by the maize weevil Sitophilus zeamais during storage. Nigerian J Expt. Biol 4 (2), 175-179. 
Adedire C O, Obembe O O, Akinkurolele R O, Oduleye O. 2011. Response of Callosobruchus maculatus (Coleoptera: Chysomelidae: Bruchinae) to extracts of cashew kernels. J Plant Dis Protect 118 (2), 75-79.

Akinkurolere R O, Sebastien B, Haoliang C, Hongyu Z. 2009. Parasitism and host location preference in Habrobracon hebetor (Hymenoptera:Braconidae): Role of refuge, choice and host instar. J Econ Entomol 102 (2), 610-615. http://dx.doi.org/10.1603/029.102.0219. PMid:19455754

Alam M Z. 1971. Pests of stored grains and other stored products and their control. Agricultural Information Service. Khamarbari, Farmgate, Dhaka-1215. p 61.

Ashamo M O, Odeyemi O O. 2001. Protection of maize against Sitophilus zeamais using seed extracts from some indigenous plants. J Plant Dis Prot 108 (3), 321-326.

Chitra E R, Xie I M, Visalakshi M M, Gour T B.1993. Impact of insecticide resistance on the biology of Rhyzopertha dominica in maize. Insect Environ 10 (1), 3-4.

lleke K D, Bulus D S. 2012. Evaluation of contact toxicity and fumigant effect of some medicinal plant and pirimiphos methyl powders against cowpea bruchid, Callosobruchus maculatus (F.) (Coleoptera: Chrysomelidae) in stored cowpea seeds. J Agricul Sci 4 (4), 279-284. http://dx.doi.org/10.5539/jas.v4n4p279

lleke K D, Odeyemi O O, Ashamo M O. 2012. Insecticidal activity of Alstonia boonei De Wild powder against cowpea bruchid, Callosobruchus maculatus (F.) (Coleoptera: Chrysomelidae) in stored cowpea seeds. Intern J Biol 4 (2), 125-131. http://dx.doi.org/10.5539/ijb.v4n2p125

Ileke K D, Olotuah O F. 2012. Bioactivity of Anacardium occidentals and Allium sativum powders and oils extracts against cowpea bruchid, Callosobruchus maculatus (F.) (Coleoptera: Chrysomelidae). Intern J Biol 4 (1), 96-103.

lleke KD, Oni M O. 2011. Toxicity of some plant powders to maize weevil, Sitophilus zeamais (Coleoptera: Curculionidae) on stored wheat grains. African J Agricul Res 6 (13), 3043-3048.

Jacobson H V. 2004. Nutritional preferences of the lesser grain borer, Rhizopertha dominica (F.) (Coleoptera: Bostrichidae) under conditions of free choice of food. J Plant Prot Res 46 (4), 359-367.

Jilani S P, Saxena A. 1990. Goat weed, Ageratum conyzoides (L.) (Asteraceae): a rice grain protectant against storage insects. J Appl Zool Res 12 (2/3), 152-156.

Kavita H N. 2004. Abiotic and biotic factors affect efficacy of chlorfenapyral for control of stored-product insect pests. J Food Prot 74 (8), 1288-1299.

Kumar H. 2009. Diatomaceous earth in Croatia. Diatom Research and Consulting Inc., Guelph, Canada. ZbornikRadova-Seminar-DDD-i-ZUPP-2009 pp 325-333.

Mbailao M, Nanadoum M, Automne B, Gabra B, Emmanuel A. 2006. Effect of six common seed oils on survival, egg lying and development of the cowpea weevil, Callosobruchus maculatus. J Biol Sci 6 (2), 420-425. http://dx.doi.org/10.3923/ibs.2006.420.425

McDonald L L, Guy R H, Speirs RD. 1970. Preliminary evaluation of new candidate materials as toxicants repellents and attractants against stored product insects. Marketing Research Report Number 882. Agricultural Research Service, U.S. Department of Agriculture, Washington $\mathrm{p} 8$.

Oni M O, lleke K D. 2008. Fumigant toxicity of four botanical plant oils on survival, egg laying and progeny development of the dried yam beetle, Dinoderus porcellus (Coleoptera: Bostrichidae). Ibadan J Agricul Res 4 (2), 31-36.

Onu I, Baba G O. 2003. Evaluation of neem products for the control of dermestid beetle on dried fish. Nigerian J Entomol 20, 105-115.

Salem S A, Abou-Ela R G, Matter M M, El-Kholy M Y. 2007. Entomocidal effect of Brassica napus extracts on two store pests, Sitophilus oryzae (L.) and Rhizopertha dominica (Fab.) (Coleoptera). J Appl Sci Res 3, 317-322. 
Schmutterer H. 1990. Properties and potential of natural pesticides from the neem tree, Azadirachta indica. Ann Rev Entomol 35, 271- 297. http://dx.doi.org/10.1146/annurev.en.35.010190.001415, PMid:2405771

Singh M Z. 1983. A study on the use of indigenous technological knowledge (ITK) for rice pest management in Assam. Insect Environ 9 (2), 91-92.

Soon L G, Bottrell D G. 1994. Neem pesticides in rice: potential and limitations. International Rice Research Institute (IRRI), Manila, Philippines p 69.

Talukdar W, Howse F. 1993. Effect of carbon dioxide rich atmosphere on storage insects and fungi. Indian J Agricul Sci 77 (11), 756-761.

Talukdar W, Howse F. 1994. Evaluation of a new insecticide formulation $\left(F_{2}\right)$ as a protectant of stored wheat, maize, and rice. J Stored Prod Res 40 (3), 317-330.

Udo I O. 2011. Potential of Zanthoxylum xanthoxyloides (Lam.) for the control of stored product insect pests. J Stored Prod Posthav Res 2 (3), 40-44.

Wright E H. 1985. Electron beam fluidized bed processing of foodstuffs. Irradiation food packaging recent devel 10 (4), 27-42. 